\title{
The Effects of Equine-assisted Activities and Therapy on Resting-state Brain Function in Attention-deficit/Hyperactivity Disorder: A Pilot Study
}

\author{
Jae Hyun $\mathrm{Yoo}^{1,2, *}$, Yunhye $\mathrm{Oh}^{3, *}$, Byongsu Jang ${ }^{4}$, Jihye Song ${ }^{5}$, Jiwon $\mathrm{Kim}^{6}$, Seonwoo Kim ${ }^{6}$, Jiyoung Lee ${ }^{7}$, \\ Hye-Yeon Shin ${ }^{7}$, Jeong-Yi Kwon ${ }^{8}$, Yun-Hee Kim ${ }^{8}$, Bumseok Jeong ${ }^{1,2}$, Yoo-Sook Joung ${ }^{3}$ \\ ${ }^{1}$ Laboratory of Clinical Neuroscience and Development, Graduate School of Medical Science and Engineering, KAIST, ${ }^{2}$ KAIST Institute \\ for Health Science and Technology, KAIST, Daejeon, ${ }^{3}$ Department of Psychiatry, Samsung Medical Center, Sungkyunkwan University \\ School of Medicine, Seoul, ${ }^{4} \mathrm{Hae}-$ sol Psychiatric Clinic, Dongtan, ${ }^{5} \mathrm{Hae}-\mathrm{sol}$ Psychiatric Clinic, Seoul, ${ }^{6}$ Samsung Biomedical Research \\ Institute, Seoul, ${ }^{7}$ Samsung Research \& Development Center, Samsung Equestrian Team, Gunpo, ${ }^{8}$ Department of Physical and Rehabilitation \\ Medicine, Samsung Medical Center, Sungkyunkwan University School of Medicine, Seoul, Korea
}

\begin{abstract}
Objective: Equine-assisted activities and therapy (EAA/T) have been used as adjunct treatment options for physical and psychosocial rehabilitation. However, the therapeutic effects on resting-state brain function have not yet been studied. The aim of this study is to investigate the effects of EAA/T on participants with attention-deficit/hyperactivity disorder (ADHD) by comparing resting-state functional magnetic resonance imaging ( $r s-f M R I)$ signals and their clinical correlates.

Methods: Ten participants with ADHD participated in a 12-week EAA/T program without any medication. Two rs-fMRIs were acquired for all participants before and after EAA/T. For estimating therapeutic effect, the regional homogeneity (ReHo) method was applied to capture the changes in the regional synchronization of functional signals.

Results: After the EAA/T program, clear symptom improvement was found even without medication. Surface-based pairwise comparisons revealed that ReHo in the right precuneus and right pars orbitalis clusters had significantly diminished after the program. Reduced ReHo in the right precuneus cluster was positively correlated with changes in the scores on DuPaul's ADHD Rating Scale-Korean version.

Conclusion: Our results indicate that $E A A / T$ is associated with short-range functional connectivity in the regions related to the default mode network and the behavioral inhibition system, which are associated with symptom improvement.
\end{abstract}

KEY WORDS: Equine-assisted therapy; Regional homogeneity; Attention deficit hyperactivity disorder; Functional magnetic resonance imaging; Resting state; Default mode network.

\section{INTRODUCTION}

Attention-deficit/hyperactivity disorder (ADHD) is considered to be the most prevalent neurodevelopmental

\footnotetext{
Received: April 21, 2016 / Revised: June 14, 2016

Accepted: August 25, 2016

Address for correspondence: Bumseok Jeong, MD, PhD Laboratory of Clinical Neuroscience and Development, Graduate School of Medical Science and Engineering, KAIST, 291 Daehakro, Yuseong-gu, Daejeon 34141, Korea

Tel: +82-42-350-4245, Fax: +82-42-350-7168

E-mail: bs.jeong@kaist.ac.kr

Yoo-Sook Joung, MD, PhD

Department of Psychiatry, Samsung Medical Center, Sungkyunkwan University School of Medicine, 81 Irwon-ro, Gangnam-gu, Seoul 06351, Korea

Tel: +82-2-3410-0930, Fax: +82-2-3410-0052

E-mail: yschoung@skku.edu

Bumseok Jeong and Yoo-Sook Joung were co-corresponding author of this article.

*These authors contributed equally to this study as co-first authors.
}

disorder, ${ }^{1)}$ with a prevalence of $3 \%$ to $7 \%$ in school-aged children. ${ }^{2)}$ The typical characteristics of ADHD are inattention and/or hyperactivity and impulsivity which cause significant impairment in social relationships and functional impairment. ${ }^{3)}$ Additionally, children with ADHD have frequently reported motor impairment, in particular, loss of balance, bilateral coordination and fine motor integration. ${ }^{4)}$ To date, pharmacologic intervention have been primary option to alleviate symptoms of ADHD. ${ }^{5-7)}$ However, there are growing body of evidences that non-pharmacological options including parental education, school-based behavioral therapy, cognitive training and physical exercise also can improve ADHD symptoms, although their effect sizes are still beyond pharmacologic treatment. ${ }^{8-10)}$

Equine-assisted activities and therapy (EAA/T) is an emerging treatment option appertain to non-pharmacologic interventions of ADHD. A recent systematic re-

(c) This is an Open-Access article distributed under the terms of the Creative Commons Attribution Non-Commercial License (http://creativecommons.org/licenses/by-nc/4.0) which permits unrestricted non-commercial use, distribution, and reproduction in any medium, provided the original work is properly cited. 
view ${ }^{11)}$ of 14 EAA/T studies have validated psychotherapeutic effectiveness that significant biopsychosocial outcomes have been achieved, such as decreased negative affect, improvement of adaptive functioning, and perceived wellness. In addition, a randomized controlled trial demonstrated that children with autism spectrum disorder who received 10-week therapeutic horseback riding showed not only significant improvement in social cognition, communication and expressive language but also irritability and hyperactivity behaviors compared to barn activity control groups. ${ }^{12)}$ Application of EAA/T on ADHD subjects have been in the beginning stage and a very few studies addressed these issues. A study with 5 ADHD participants did demonstrate improvement in the domains of behavior, quality of life, and motor performance after 16 sessions of EAA/T. ${ }^{13)}$ In addition, Jang and colleagues reported that 12-week EAA/T resulted mitigation of social problem and motor incoordination as well as symptom improvement by ADHD rating scale. ${ }^{14)}$ Although its beneficial effects is thought to be mediated by facilitating development of motor skills, coordination and sensory processing, ${ }^{15)}$ feelings of mastering and self-efficacy and selfconfidence, ${ }^{16)}$ exact neural mechanism of the EAA/T has not been proved yet.

Resting-state functional magnetic resonance imaging (rs-fMRI) is a tool that could display the intrinsic functional architecture of the brain. ${ }^{17)}$ Among them, the default mode network (DMN) are neural system consisting of medial prefrontal cortex, precuneus/posterior cingulate cortex and inferior parietal lobule, and typically more active during rest than during active task performance. ${ }^{18)}$ There are growing body of evidences that aberrant connectivity between DMN and other resting state networks have a critical role in ADHD pathology. ${ }^{5,17,19,20)}$ Hence, altered spontaneous activity and connectivity within DMN have been reported in ADHD studies. ${ }^{6,21)}$ Functional connectivity between DMN and task-positive regions was critically related to poor task performance, ${ }^{20,22,23)}$ which might be relevant to clinical manifestations in ADHD. Therefore, suggested mechanism for symptom improvement after EAA/T might be related to functional change of the DMN.

We have previously reported the effectiveness of 12week EAA/T for improving core ADHD symptoms as well as reducing theta/beta ratio in Pz area. ${ }^{14)}$ However, relationship between symptom improvement and changes within resting functional network have not been fully investigated. In this pilot study, we hypothesized that therapeutic effect of EAA/T would be reflected as a change in spontaneous neural activity during resting state, especially in the regions related to the DMN. To test our hypotheses, we analyzed rs-fMRI data acquired from 10 children with ADHD who were participated in whole 12-week EAA/T program. Therapeutic effect was determined from pairwise comparison between regional homogeneity ( $\mathrm{ReHo}$ ) calculated from pre- and post-EAA/T state.

\section{METHODS}

\section{Participants}

A total of 22 participants ( 21 boys and 1 girl) were recruited from the Child and Adolescent Psychiatry Service Unit at Samsung Medical Center, Seoul, Republic of Korea. They received a diagnosis of ADHD according to the American Psychiatric Association's Diagnostic and Statistical Manual of Mental Disorders, 4th edition, text revision (DSM-IV-TR). ${ }^{24)}$ The Korean Kiddie-Schedule for Affective Disorders and Schizophrenia-Present and Lifetime Version were administered by two psychiatrists trained in the use of this interview in order to confirm ADHD. Two children who were taking methylphenidate or atomoxetine within 90 days of baseline were excluded. Among 20 participants who completed the 12 weeks of EAA/T, 10 boys were excluded from the rs-fMRI analysis because of refusal of scanning, dental braces and excessive head motion. Finally, 10 children ( 9 boys and 1 girl) who received MRI studies two weeks before and after the EAA/T were included in the current analysis. The mean age of the 10 ADHD participants was $8.3 \pm 1.3$ years, and mean intelligence quotient (IQ) measured with the Korean version of the Wechsler Intelligence Scale for Children-fourth edition was $98.5 \pm 17.9$. There were no significant demographic differences between the completed and excluded participants. This study protocol was approved by the institutional review board of Samsung Medical Center, Seoul, Republic of Korea (IRB File No: 2012-12-049-001). Signed informed consent was obtained from all participants and their parents.

\section{Design}

The participants received an initial comprehensive evaluation (visit 1) to determine study eligibility. They were given a 30-day period prior to visit 2. During visit 2, Rating scales including DuPaul's ADHD Rating Scale-Korean version (K-ARS), ${ }^{24}$ The Clinical Global ImpressionGlobal Improvement (CGI-I), and the Developmental Coordination Disorder Questionnaire (DCDQ) ${ }^{25}$ ) were administered to evaluate symptom severity, improvement and motor coordination ability, respectively. The EAA/T 
started within 2 weeks after visit 2 and continued for 12 weeks. After the EAA/T, all study scales were re-administered (visit 3) within 2 weeks. All participants were rated by the same investigator with open label. Any psychotropic medications were not permitted after initiating the EAA/T program to reduce the effect of confounding factors. ${ }^{8)}$

\section{EAA/T Program}

The EAA/T was based on a psycho-exercise program to improve attention and inhibit impulsivity. The program was held twice per week for 12 weeks (total of 24 sessions) in indoor riding arena. Each lesson included horsemanship activities such as grooming, tacking and feeding, and riding activities following their progressions, total an hour long lesson. Detailed protocol of the sessions over the 12 weeks is descried in Table 1 . The child was accompanied by his parents on each session, wore protective equipment (helmet, boots, stirrups, safety vest, and gloves) and was accompanied by two trained side walkers and a leader for safety issue. The horses/ponies used for our EAA/T program, had been well trained to engage in hippotherapy, and had much experience of therapeutic riding sessions. They were assigned to the classes with an assignment guideline: no more than two consecutive classes, no longer than two hour long work.

EAA/T program for ADHD were ran by two hippotherapy clinical specialists (HPCS). They took hipppotherapy courses level I and II by American Hippotherapy Association and passed the exam for the HPCS. They also hold PATH Intl. registered therapeutic riding instructor certificate. There was also one more PATH Intl. registered therapeutic riding instructor helping out the program. All three of them holds Korean therapeutic riding instructor certificate issued by Korean Ministry of Agriculture, Food and Rural Affairs.

\section{Imaging Data Acquisition}

MRI scans were performed for each participant before (visit 2) and after (visit 3) the intervention. MRI safety was checked before the scans by participants and their parents using a checklist. Each participant's head was stabilized with cushions and taped to help minimize movement. The whole scanning process was monitored by the study's authors and a parent inside the scanning room. Using a Philips 3T Achieva scanner (Philips Medical Systems, Best, The Netherlands), high-resolution T1 images were acquired with the following parameters: repetition time $(\mathrm{TR})=9.9 \mathrm{~ms}$, echo time $(\mathrm{TE})=4.6 \mathrm{~ms}$, field of view $(\mathrm{FoV})=240 \mathrm{~mm}$, voxel size $=0.5 \times 0.47 \times 0.47 \mathrm{~mm}$, and flip angle $=8^{\circ}$. Next, rs-fMRIs that covered the whole brain were also acquired using echo planar imaging sequences $(\mathrm{TR}=3,000 \mathrm{~ms}, \mathrm{TE}=35 \mathrm{~ms}, \mathrm{FoV}=220 \mathrm{~mm}$, voxel size $=1.7 \times 1.7 \times 4.0 \mathrm{~mm}$, and flip angle $=90^{\circ}$ ).

\section{Data Preprocessing}

The MRI data were preprocessed with the FMRIB Software Library (FSL, www.fmrib.ox.ac.uk/fsl) version 5.0, Analysis of Functional NeuroImages (http://afni.nimh. nih.gov/afni/), and the FreeSurfer image analysis suite (http://surfer.nmr.mgh.harvard.edu/) version 5.3. Because our participants had separate $\mathrm{T} 1$ and resting functional magnetic resonance (fMR) images before and after the $\mathrm{EAA} / \mathrm{T}$, the data from each time point were separately processed in the following steps. First, a high-resolution T1 image of each participant was reconstructed to the cortical surface using methods based on the FreeSurfer processing pipeline: registration to the Talairach atlas, bias field correction, skull stripping, intensity normalization, surface modeling, and spherical mapping and registration. ${ }^{23,26-28)}$ Second, slice timing correction, deobliquing, and motion correction were sequentially applied to the fMR data in each participant's space. The whole image was normalized by mean intensity and scaled by a factor of 10,000, and linear and quadratic trends from each signal were removed. Next, the each fMR image was co-registered with the T1 images using a transformation

Table 1. Equine-assisted activities and therapy program schedule for 12 weeks

\begin{tabular}{|c|c|c|c|}
\hline Session & Horsemanship skill (duration) & Hippotherapy (duration) & Aims \\
\hline $\begin{array}{l}\text { 1-6th } \\
\text { Adaptation } \\
\text { period }\end{array}$ & $\begin{array}{l}\text { Grooming, greeting, main security rules, } \\
\text { child's use basic nomenclature of } \\
\text { grooming technique, praising ( } 40 \text { min) }\end{array}$ & Leading, riding (20 min) & $\begin{array}{l}\text { Facilitate emotional communication and } \\
\text { sense of closeness }\end{array}$ \\
\hline $\begin{array}{l}\text { 7-19th } \\
\text { Learning } \\
\text { period }\end{array}$ & $\begin{array}{l}\text { Master grooming, learn tacking, bridling } \\
(30 \mathrm{~min})\end{array}$ & $\begin{array}{l}\text { Learn riding basic elements (hold } \\
\text { the reign, mounting, dismounting, } \\
\text { walk, trot) ( } 30 \mathrm{~min} \text { ) }\end{array}$ & $\begin{array}{l}\text { Inhibit hyperactivity, complete task } \\
\text { requiring concentration, improving } \\
\text { balance and coordination on the horse }\end{array}$ \\
\hline $\begin{array}{l}\text { 20-24th } \\
\text { Enhance skill } \\
\text { period }\end{array}$ & $\begin{array}{l}\text { Master skill for grooming, tacking, } \\
\text { bridling (20 min) }\end{array}$ & $\begin{array}{l}\text { Master basic skill, running the full } \\
\text { course }\end{array}$ & $\begin{array}{l}\text { Repetitive training, improve self-regulation } \\
\text { skill, self-confidence, and sense of } \\
\text { accomplishment }\end{array}$ \\
\hline
\end{tabular}


matrix created from boundary-based registration. Finally, 5 major components from time course of white matter and cerebrospinal fluid were regressed out from the fMRI data using principal component analysis. This processing method can significantly reduce physiological noise as well as noise from other sources. ${ }^{29,30)}$

\section{Estimating ReHo and the Analysis Process}

Zang et $a l .{ }^{31)}$ introduced the concept of ReHo, which targets connectivity at the local level by measuring the regional similarity of the time series within surrounding voxels with Kendall's coefficient of concordance. This approach gives us a critical opportunity to not only investigate the spontaneous neuronal activity, but also explore the short-range connectivity at local level, without the need to consider any a priori hypotheses. ${ }^{32}$ To estimate ReHo, the preprocessed $\mathrm{fMR}$ data were filtered on a low-frequency band between 0.009 and $0.1 \mathrm{~Hz}$ and then resampled to $3 \mathrm{~mm}$ isotropic voxels without applying spatial smoothing. The ranks of each voxel in each TR were computed, and Kendall's coefficient of concordance was estimated within 1 target voxel and 26 surrounding voxels. The calculated ReHo data were sampled along the middle of the gray and white matter boundary and projected into the vertices of each participant's surface. Surface alignment of the functional signal has a number of advantages: it can reduce not only the inter-participant variability caused by individual cortical folding patterns, ${ }^{27,33)}$ but also the substantial spread of activation along distant regions when spatial smoothing is applied. ${ }^{34)}$

We conducted pairwise registration of the pre- and post-EAA/T fMRI data into the surface. Data on each individual surface were also moved to a common spherical surface, called 'fsaverage', and spatially smoothed with a $5 \mathrm{~mm}$ full-width at half-maximum Gaussian kernel. Finally, ReHo was transformed to a $\mathrm{Z}$ score in the surface model to compute group statistics. We estimated the effect of EAA/T using a general linear model while controlling for the effects of participants' age and IQ. In our analysis,

Table 2. Clinical characteristics of the subjects

\begin{tabular}{cccc}
\hline Variable & Pre-EAA/T & Post EAA/T & t-statistics \\
\hline K-ARS, total & $31.4 \pm 9.7$ & $16.5 \pm 6.8$ & $4.00^{*}$ \\
Inattention score & $16.1 \pm 5.0$ & $10.3 \pm 3.4$ & $3.03^{*}$ \\
Hyperactivity score & $15.3 \pm 6.1$ & $6.2 \pm 4.3$ & $3.85^{*}$ \\
DCDQ score & $60.7 \pm 6.9$ & $60.1 \pm 8.9$ & 0.17 \\
\hline
\end{tabular}

$E A A / T$, equine-assisted activities and therapy; K-ARS, Dupaul's ADHD Rating Scale-Korean version; DCDQ, Developmental Coordination Disorder Questionnaire. ${ }^{*} p<0.01$. significant clusters were defined by the following three criteria: first, clusters that met the cluster-forming threshold of $p<0.0001$; second, clusters that consisted of more than ten voxels after 10,000 Monte-Carlo z statistics simulation; and third, $p<0.05$ for each cluster after a two-tailed test and correction for both hemispheric tests. Finally, we attempted to estimate how well cluster-wise ReHo changes reflected symptom improvement in each participant. Because initial disease severity and ReHo varied by participant, we tested correlation with the percent changes in both measures. Because our sample size was small $(n=10)$, Spearman's rank-order correlation was used for correlation analysis. Correlation analyses were performed with in-house code written in MATLAB software (2013a; MathWorks, Inc., Natick, MA, USA).

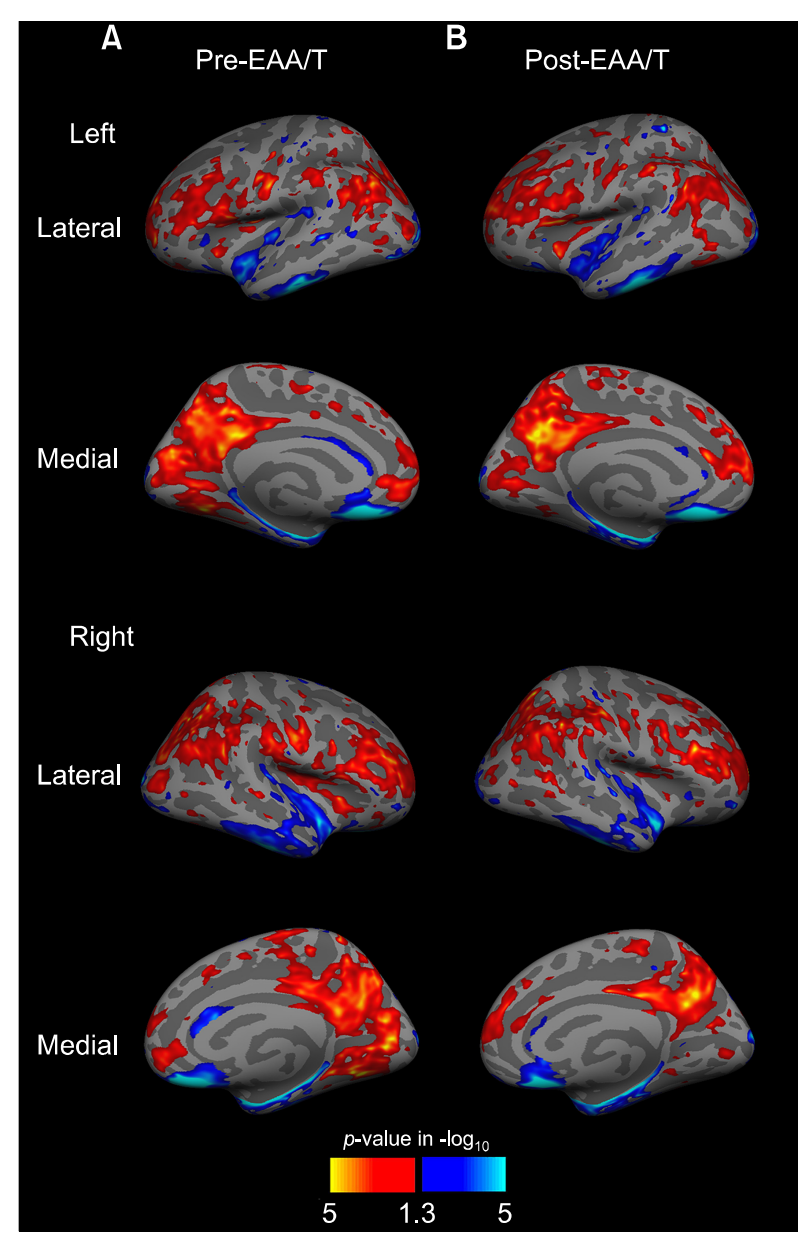

Fig. 1. Results of regional homogeneity ( $\mathrm{ReHo}$ ) statistical maps preand post-EAA/T. Red and yellow indicate higher ReHo, and blue and cyan indicate lower ReHo than average on the whole surface in one sample t-test. The surface overlay displays the uncorrected $p$-value in - $\log _{10}$.

$E A A / T$, equine-assisted activities and therapy. 


\section{RESULTS}

The clinical characteristics of the participants are presented in Table 2. Scores on the K-ARS overall and on its two subscales decreased after 12 weeks of the EAA/T. Symptom improvement was also revealed in the CGI-I ratings; six of the ten participants were rated as much improved, and the other four were found to have minimal improvement. However, we could not find significant differences in the DCDQ scores after treatment.

Surface-projected maps of the ReHo were built using a one-sample $t$-test at each pre- and post-EAA/T time point (Fig. 1). Comparing average ReHo on the whole brain surface revealed that higher ReHo was prominent in the nodes of the DMN, including the posterior cingulate cortex, precuneus, medial prefrontal cortex, and inferior frontal lobe. In contrast, the superior and inferior parts of the tem-

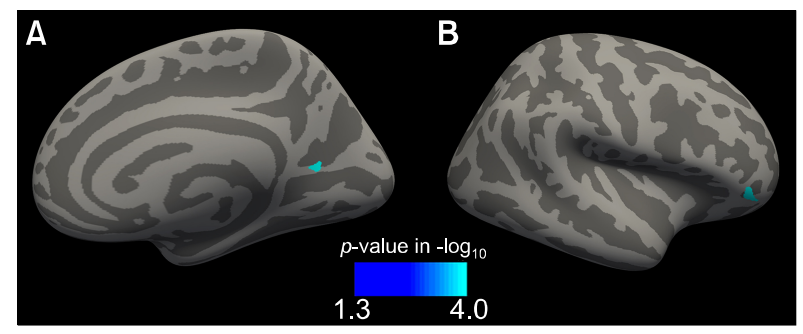

Fig. 2. Statistical maps of a cluster that displayed reduced regional homogeneity in the pairwise comparison. Clusters were found at the right (A) precuneus and (B) pars orbitalis surface. The surface overlay displays the family-wise corrected $p$-values calculated from 10,000 simulations of Monte-Carlo Z statistics.

A

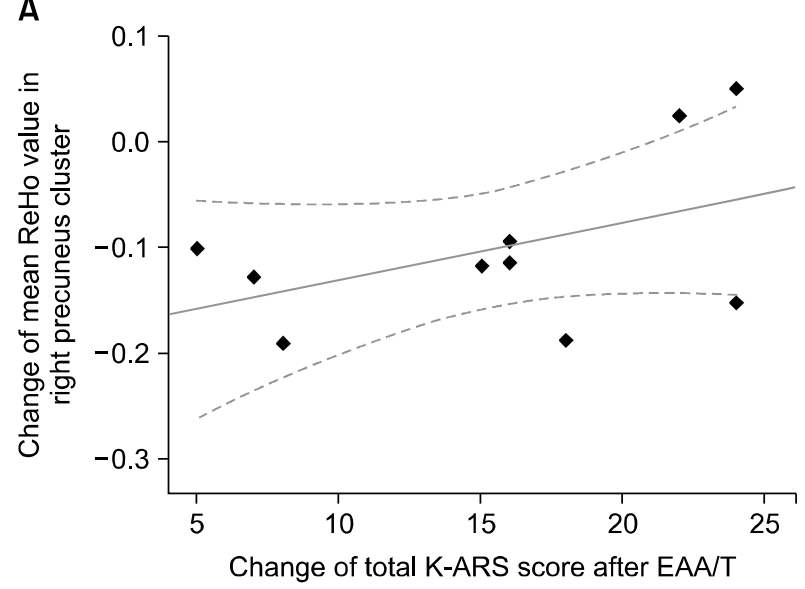

poral gyrus and parahippocampal region showed less ReHo than did other brain areas.

From the pairwise comparisons of the ReHo, significant effects of EAA/T were found in two clusters, in the right precuneus and pars orbitalis $(p<0.001$ and $p$ $<0.001$ after multiple comparison correction, respectively; Fig. 2); both showed reduced ReHo after the EAA/T (Table 3). Among them, the mean ReHo change in the right precuneus cluster was significantly correlated with lower total K-ARS scores after the EAA/T (Spearman's Rho $=-0.809, p=0.031$; Fig. 3). Additionally, marginal correlations were also found in the mean ReHo in the pars orbitalis cluster and in the changes in DCDQ scores (Spearman's Rho $=0.726, p=0.065$ ).

\section{DISCUSSION}

In this study, a 12-week EAA/T program induced significant changes of local connectivity in ADHD participants. Two distinct clusters were identified from the pair-

Table 3. Summary of the brain areas that showed reduced ReHo after EAA/T

\begin{tabular}{lccc}
\hline \multicolumn{1}{c}{ Region } & $\begin{array}{c}\text { Peak location } \\
\text { (MNI coordinates) }\end{array}$ & Vertices & $\begin{array}{c}\text { Maximum } \\
\text { Z score }\end{array}$ \\
\hline Right precuneus & $22.0,-59.7,11.7$ & 64 & $-5.712^{*}$ \\
Right pars orbitalis & $42.8,38.3,-6.1$ & 27 & $-4.658^{*}$ \\
\hline
\end{tabular}

ReHo, regional homogeneity; EAA/T, equine-assisted activities and therapy; MNI, Montreal Neurological Institute. ${ }^{*} p<0.001$.

B

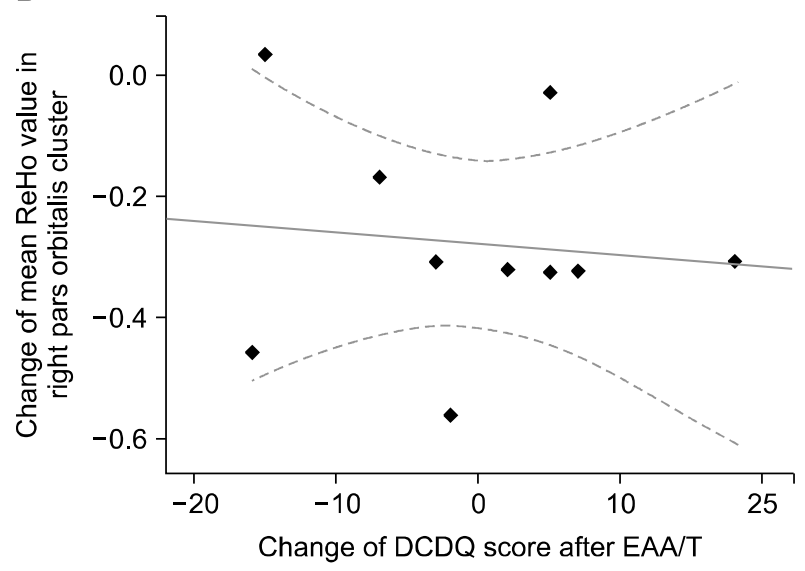

Fig. 3. Scatter plots illustrating the relationship between ReHo changes and clinical correlates after EAA/T. (A) Reduced ReHo in the precuneus cluster was positively correlated with improved total K-ARS score (Spearman's Rho $=-0.809, p=0.03$ ). (B) Marginal correlation was found between reduced ReHo in the pars orbitalis and increased DCDQ score (Spearman's Rho=0.726, $p=0.065$ ).

ReHo, regional homogeneity; EAA/T, equine-assisted activities and therapy; K-ARS, Dupaul's ADHD Rating Scale-Korean version; DCDQ, Developmental Coordination Disorder Questionnaire. 
wise comparison, one in the right precuneus and the other in the right pars orbitalis surface. The ReHo value in the right precuneus cluster showed significant correlation with improved total K-ARS scores, and that of pars orbitalis also marginally correlated with motor coordination changes measured by the DCDQ. Although these results were yielded from a small group of ADHD participants, the current study is the first report to reveal the effects of $\mathrm{EAA} / \mathrm{T}$ by investigating the changes in resting functional connectivity and their behavioral correlates.

Surface ReHo map in current study revealed that higher regions were located within the posterior DMN and surrounding clusters, and lower values related to regions related to the task-associated network, which is consistent with previous results. ${ }^{14,35)}$ Intriguingly, EAA/T resulted alteration of distribution patterns in the clusters near the posterior DMN; specifically, the number of clusters diminished after treatment and converged to a single cluster throughout the precuneus and the posterior cingulate. This finding is generally consistent with the results from An et $a l{ }^{36)}$ that higher baseline ReHo within the parietal and lingual gyrus in a placebo group had diminished after methylphenidate treatment.

The pairwise comparisons revealed a main effect of 12 weeks of EAA/T in the precuneus and pars orbitalis clusters. The precuneus is known to not only be a key member of the DMN, which plays a role in mental imagery and self-referential thoughts, but also related to episodic memory retrieval and visuospatial processing. ${ }^{37)}$ The precuneus has drawn particular attention in ADHD participants because aberrant connectivity in that region has been consistently reported in previous resting-state and task-related MRI studies. ${ }^{5,6,38)}$ One study revealed that smaller deactivations in the precuneus during tasks were associated with longer response times, which is frequently reported in ADHD participants as momentary lapses. ${ }^{39)}$ Although we could not compare a treatment-naïve group with typically developing children, considering the findings of An and colleagues, ${ }^{36)}$ the decreased ReHo in this region possibly reflected the normalization of up-regulated $\mathrm{ReHo}$ or the reorganization of the foci of regional connectivity after the EAA/T. This rough assumption is supported by correlations with the K-ARS scores, which reflected that participants who had greater decreases in ReHo in the precuneus cluster also showed more symptom improvement.

Another significant ReHo change was identified in clusters located at the right pars orbitalis, a part of the inferior frontal gyrus (IFG). Previous studies revealed that
IFG plays a critical role in behavioral inhibition ${ }^{40-42)}$ and executive motor control, ${ }^{26)}$ which is thought to be impaired in ADHD ${ }^{28)}$ Pharmacologic treatment improved inhibitory control performance, together with the normalization of IFG activity and its functional interaction with surrounding regions such as ventral prefrontal cortex. ${ }^{27)}$ Previous connectivity study reported that right IFG had a negative correlation with the ventromedial part of the prefrontal cortex, which is a crucial node of the anterior DMN ${ }^{5)}$ In addition, stringent antiphase relationship related with greater behavioral performance in a number of previous studies. ${ }^{20,23,39)}$ Regarding quiescent nature of task-positive network during resting-state, therapeutic effect of EAA/T on inhibitory control might be mediated by reducing ReHo in the right IFG.

Although the current pilot study is the first to demonstrate the effects of EAA/T on functional changes in brain regions, a number of limitations should be taken into account. First, our study included only a subset of ADHD participants who finished the 12-weeks of EAA/T. Comparison with the typically developing children would be needed in the future, however, our results first delineated changes in functional connectivity by EAA/T. Second, we only analyzed resting-state data, and thus, the direct effects of EAA/T on task-related performance such as attention, emotion, and cognition could not be estimated. Investigating those effects requires task-specific fMRI designs for each domain. Lastly, long-term effects could not be examined from a single 12-week EAA/T session. For equine therapy to be accepted as an adjunct therapeutic option for ADHD, additional studies with larger samples, controlled designs, and purposeful fMRI sessions are necessary.

In conclusion, the current study found that $\mathrm{EAA} / \mathrm{T}$ in ADHD participants is associated with subsequent resting-state functional signal changes in the right precuneus and pars orbitalis clusters. Furthermore, the functional changes in the precuneus were significantly correlated with clinical improvement, which suggests that dysfunctional modulation in the DMN partly might be associated with symptom improvement of ADHD participants. Although limited sample size and design masked generalizability, this study showed a potential mechanism for how EAA/T has a positive influence on ADHD.

\section{- Acknowledgments}

On behalf of all authors, the corresponding author states that there is no conflict of interest. This study was conducted by Special Reserve Fund of Korea Racing 
Authority and was partly supported by a grant from the KAIST Future Systems Healthcare Project from the Ministry of Education, Science and Technology (N01150030 to B Jeong) and KAIST Venture Research Program for Graduate \& Ph.D students.

\section{REFERENCES}

1. Braaten EB, Rosén LA. Self-regulation of affect in attention deficit-hyperactivity disorder (ADHD) and non-ADHD boys: differences in empathic responding. J Consult Clin Psychol 2000;68:313-321.

2. Polanczyk G, de Lima MS, Horta BL, Biederman J, Rohde LA. The worldwide prevalence of ADHD: a systematic review and metaregression analysis. Am J Psychiatry 2007; 164:942-948.

3. Spencer TJ, Biederman J, Mick E. Attention-deficit/hyperactivity disorder: diagnosis, lifespan, comorbidities, and neurobiology. J Pediatr Psychol 2007;32:631-642.

4. Cho H, Ji S, Chung S, Kim M, Joung YS. Motor function in school-aged children with attention-deficit/hyperactivity disorder in Korea. Psychiatry Investig 2014;11:223-227.

5. Uddin LQ, Kelly AM, Biswal BB, Margulies DS, Shehzad $\mathrm{Z}$, Shaw $\mathrm{D}$, et al. Network homogeneity reveals decreased integrity of default-mode network in ADHD. J Neurosci Methods 2008;169:249-254.

6. Cao Q, Zang Y, Sun L, Sui M, Long X, Zou Q, et al. Abnormal neural activity in children with attention deficit hyperactivity disorder: a resting-state functional magnetic resonance imaging study. Neuroreport 2006;17:1033-1036.

7. Jung C, Choi S, Jeong S, Song C, Seo W, Chung US, et al. Multicenter, open-label study to evaluate the effects of methylphenidate-OROS(Concerta $\AA$ ) on cognitive functions in children with attention deficit hyperactivity disorder. Clin Psychopharmacol Neurosci 2007;5:31-37.

8. Evans SW, Owens JS, Bunford N. Evidence-based psychosocial treatments for children and adolescents with attention-deficit/hyperactivity disorder. J Clin Child Adolesc Psychol 2014;43:527-551.

9. Fabiano GA, Schatz NK, Aloe AM, Chacko A, ChronisTuscano A. A systematic review of meta-analyses of psychosocial treatment for attention-deficit/hyperactivity disorder. Clin Child Fam Psychol Rev 2015;18:77-97.

10. Pontifex MB, Saliba BJ, Raine LB, Picchietti DL, Hillman $\mathrm{CH}$. Exercise improves behavioral, neurocognitive, and scholastic performance in children with attention-deficit/ hyperactivity disorder. J Pediatr 2013;162:543-551.

11. Selby A, Smith-Osborne A. A systematic review of effectiveness of complementary and adjunct therapies and interventions involving equines. Health Psychol 2013;32: 418-432.

12. Pan CY, Chang YK, Tsai CL, Chu CH, Cheng YW, Sung MC. Effects of physical activity intervention on motor proficiency and physical fitness in children with ADHD: an exploratory study. J Atten Disord 2014. doi: 10.1177/ 1087054714533192. [Epub ahead of print]

13. Cuypers K, De Ridder K, Strandheim A. The effect of therapeutic horseback riding on 5 children with attention deficit hyperactivity disorder: a pilot study. J Altern Complement Med 2011;17:901-908.

14. Chao-Gan Y, Yu-Feng Z. DPARSF: A MATLAB toolbox for "Pipeline" data analysis of resting-state fMRI. Front Syst Neurosci 2010;4:13.
15. Herrero P, Asensio A, García E, Marco A, Oliván B, Ibarz $\mathrm{A}$, et al. Study of the therapeutic effects of an advanced hippotherapy simulator in children with cerebral palsy: a randomised controlled trial. BMC Musculoskelet Disord 2010;11:71.

16. Hauge H, Kvalem IL, Berget B, Enders-Slegers MJ, Braastad BO. Equine-assisted activities and the impact on perceived social support, self-esteem and self-efficacy among adolescents - an intervention study. Int J Adolesc Youth 2014;19:1-21.

17. Castellanos FX, Proal E. Large-scale brain systems in ADHD: beyond the prefrontal-striatal model. Trends Cogn Sci 2012;16:17-26.

18. Buckner RL, Andrews-Hanna JR, Schacter DL. The brain's default network: anatomy, function, and relevance to disease. Ann N Y Acad Sci 2008;1124:1-38.

19. Choi J, Jeong B, Lee SW, Go HJ. Aberrant development of functional connectivity among resting state-related functional networks in medication-naïve ADHD children. PLoS One 2013;8:e83516.

20. Kelly AM, Uddin LQ, Biswal BB, Castellanos FX, Milham MP. Competition between functional brain networks mediates behavioral variability. Neuroimage 2008;39:527537.

21. Zang YF, He Y, Zhu CZ, Cao QJ, Sui MQ, Liang M, et al. Altered baseline brain activity in children with ADHD revealed by resting-state functional MRI. Brain Dev 2007:29:83-91.

22. Christakou A, Murphy CM, Chantiluke K, Cubillo AI, Smith $\mathrm{AB}$, Giampietro $\mathrm{V}$, et al. Disorder-specific functional abnormalities during sustained attention in youth with Attention Deficit Hyperactivity Disorder (ADHD) and with autism. Mol Psychiatry 2013;18:236-244.

23. Fassbender C, Zhang H, Buzy WM, Cortes CR, Mizuiri D, Beckett L, et al. A lack of default network suppression is linked to increased distractibility in ADHD. Brain Res 2009;1273:114-128.

24. So YK, Noh JS, Kim YS, Ko SG, Koh YJ. [The reliability and validity of Korean Parent and Teacher ADHD Rating Scale]. J Korean Neuropsychiatr Assoc 2002;41:283-289. Korean.

25. Schoemaker MM, Flapper B, Verheij NP, Wilson BN, Reinders-Messelink HA, de Kloet A. Evaluation of the Developmental Coordination Disorder Questionnaire as a screening instrument. Dev Med Child Neurol 2006;48: 668-673.

26. Tanji J, Hoshi E. Role of the lateral prefrontal cortex in executive behavioral control. Physiol Rev 2008;88:37-57.

27. Peterson BS, Potenza MN, Wang Z, Zhu H, Martin A, Marsh R, et al. An FMRI study of the effects of psychostimulants on default-mode processing during Stroop task performance in youths with ADHD. Am J Psychiatry 2009; 166:1286-1294.

28. Aron AR, Poldrack RA. The cognitive neuroscience of response inhibition: relevance for genetic research in attention-deficithyperactivity disorder. Biol Psychiatry 2005; 57:1285-1292.

29. Behzadi Y, Restom K, Liau J, Liu TT. A component based noise correction method (CompCor) for BOLD and perfusion based fMRI. Neuroimage 2007;37:90-101.

30. Chai XJ, Castañón AN, Ongür D, Whitfield-Gabrieli S. Anticorrelations in resting state networks without global signal regression. Neuroimage 2012;59:1420-1428.

31. Zang Y, Jiang T, Lu Y, He Y, Tian L. Regional homogeneity approach to fMRI data analysis. Neuroimage 2004;22:394- 
400

32. Zhang Z, Lu G, Zhong Y, Tan Q, Chen H, Liao W, et al. fMRI study of mesial temporal lobe epilepsy using amplitude of low-frequency fluctuation analysis. Hum Brain Mapp 2010;31:1851-1861.

33. Argall BD, Saad ZS, Beauchamp MS. Simplified intersubject averaging on the cortical surface using SUMA. Hum Brain Mapp 2006;27:14-27.

34. Jo HJ, Lee JM, Kim JH, Shin YW, Kim IY, Kwon JS, et al. Spatial accuracy of fMRI activation influenced by volume- and surface-based spatial smoothing techniques. Neuroimage 2007;34:550-564.

35. Zhu Y, Gao B, Hua J, Liu W, Deng Y, Zhang L, et al. Effects of methylphenidate on resting-state brain activity in normal adults: an fMRI study. Neurosci Bull 2013;29:16-27.

36. An L, Cao XH, Cao QJ, Sun L, Yang L, Zou QH, et al. Methylphenidate normalizes resting-state brain dysfunction in boys with attention deficit hyperactivity disorder. Neuropsychopharmacology 2013;38:1287-1295.

37. Cavanna AE, Trimble MR. The precuneus: a review of its functional anatomy and behavioural correlates. Brain 2006;129:564-583.

38. Castellanos FX, Margulies DS, Kelly C, Uddin LQ, Ghaffari $\mathrm{M}$, Kirsch A, et al. Cingulate-precuneus interactions: a new locus of dysfunction in adult attention-deficit/hyperactivity disorder. Biol Psychiatry 2008;63:332-337.

39. Weissman DH, Roberts KC, Visscher KM, Woldorff MG. The neural bases of momentary lapses in attention. Nat Neurosci 2006;9:971-978.

40. Aron AR, Fletcher PC, Bullmore ET, Sahakian BJ, Robbins TW. Stop-signal inhibition disrupted by damage to right inferior frontal gyrus in humans. Nat Neurosci 2003;6: 115-116.

41. Aron AR, Robbins TW, Poldrack RA. Inhibition and the right inferior frontal cortex. Trends Cogn Sci 2004;8: 170-177.

42. McNaughton N, Corr PJ. A two-dimensional neuropsychology of defense: fear/anxiety and defensive distance. Neurosci Biobehav Rev 2004;28:285-305. 